Errno::ENOENT: No such file or directory @ rb_sysopen - main.scss

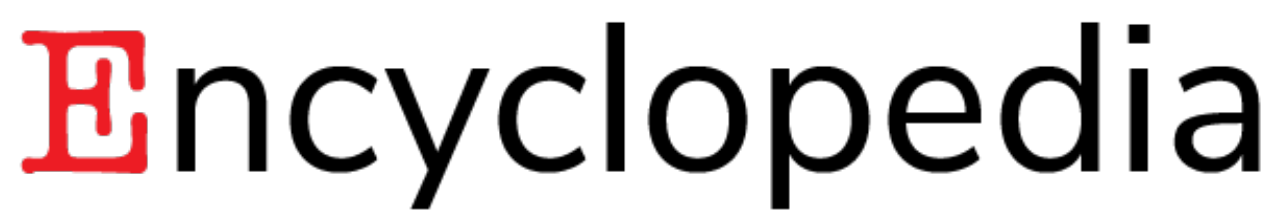

\title{
Windows-based computational algorithms for 1D MPC and thin-film analysis
}

Created by: Mohammad Nur E Alam

Revised by: Mikhail Vasiliev

Version received: 9 April 2020

check for updates

The development of magnetic photonic crystals (MPC) has been a rapidly evolving research area since the late 1990s. Magneto-optic (MO) materials and the techniques for their characterization have also continually undergone functional and property-related improvements. MPC Optimization is a feature-rich Windows software application designed to enable researchers to analyse the optical and magneto-optical spectral properties of multilayers containing gyrotropic constituents. A set of computational approaches, and a custom software package have been described, designed to enable the design and optimization of 1D magnetic photonic crystals in terms of the achievable combinations of Faraday rotation, transmission, and reflection spectra.

\section{Table of Content [Hide]}

The one-dimensional MPCs have been reported to be applicable for various existing and emerging new nanotechnological device applications, due to the functionality of Faraday rotation enhancement and also their tunable spectral properties. Many research groups have focused on optimizing the design and features of one-dimensional (1D) MPC structures using various software packages (most of them are quite complex to use and are not freely available online for a wide range of users). Since our group started working with garnet materials and garnet synthesis, the development of a new MPC optimization program has originated in 2005. After that, till 2019, this new software was continually tested in-house, and it has finally been realised that potentially a very large group of materials science professionals, especially the younger scientists who have recently started their research careers, can readily benefit from using this newly developed and freely available software package. Here, we would like to let the world's researchers discover our MPC optimization software and also would like to open the doors for their valuable feedback.

The required solution code for this MPC optimization program has been written by using Microsoft Visual Studio 2003 .NET Professional, by Dr Mikhail Vasiliev, using Microsoft Managed C++ .NET; multiple code changes and program feature additions have been applied in-between 2005-2019. During this time-frame, the development of the algorithms and the code features of this program have been discussed within our research group (Prof Kamal Alameh and Dr Mohammad Nur-E-Alam, and further program developments were greatly influenced and experimentally tested through our work on the multiple materials-related projects. Figure 1 is reproduced from the published article in MDPI Technologies $^{[1]}$ which shows the graphical snapshot of the front-panel controls of MPC Optimization software. The details about the program code of this MPC software, and its features are outlined in Ref.[1] together with the installation instructions. This is a very easy-to-use installation software, which is enabled by running the installer (.msi) file, supplied within the .zip archived folder used for the program redistribution. However, there is a necessary prerequisite package to install this program, which is the Microsoft .NET Framework 1.1, which must be installed on any Windows machine prior to running the MPC Optimization installer. The .NET 1.1 Framework installation file (dotnetfx.exe, available also from Microsoft) is also supplied within the archived folder file used to redistribute MPC Optimization software package. 


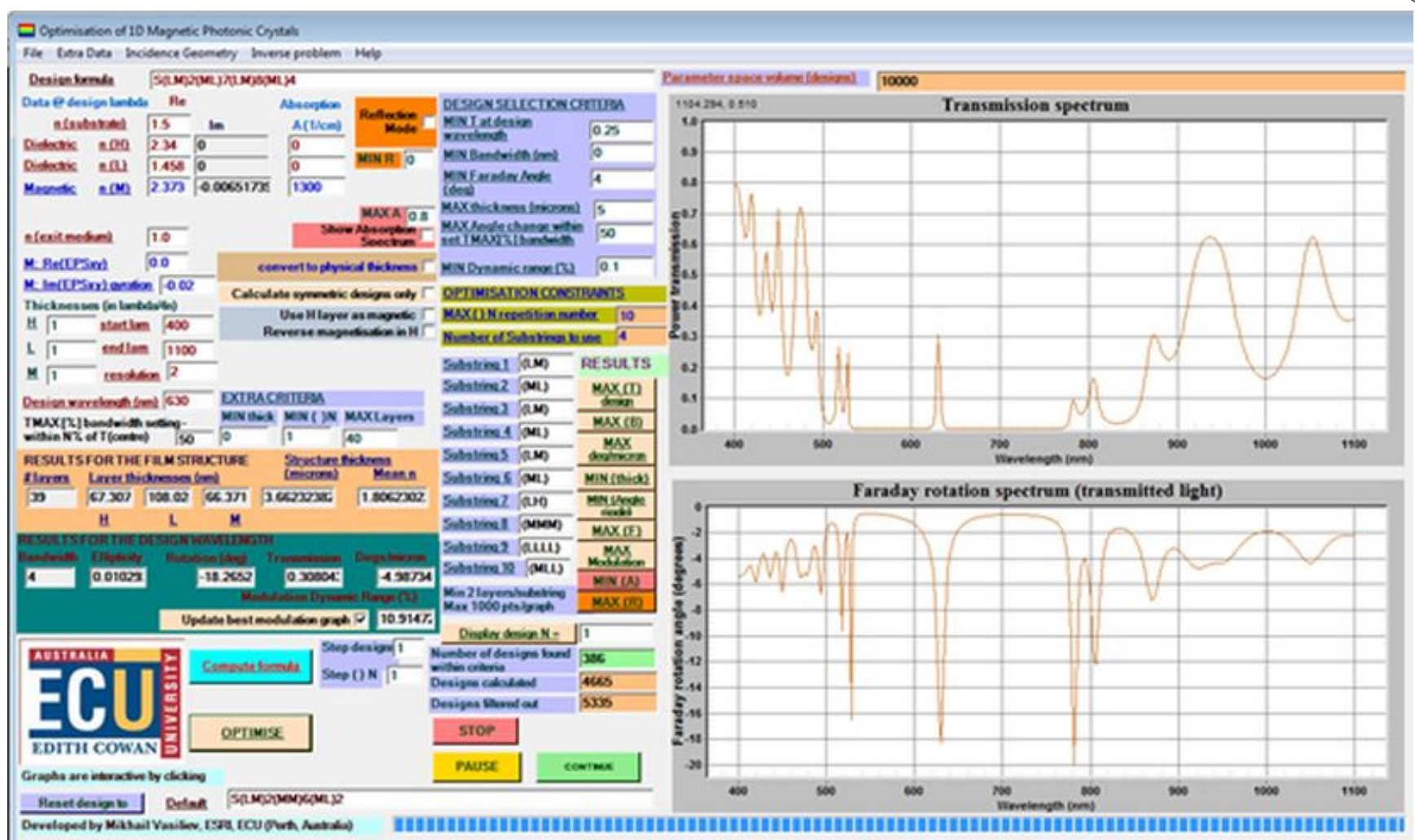

Figure 1: A graphical snapshot of the front-panel controls of the MPC Optimization software, and the features implemented within MPC Optimization, showing a sample optimization result.

This MPC software package also provides the features of characterising the low-absorptive semitransparent (single layer) thin-film materials. It is possible to fit the measured transmission spectrum of either new or previously known thin-film materials with the model-predicted transmission spectrum, thus allowing to calculate the actual best-fitted film thickness, and to derive the unknown optical parameters (e.g. the absorption coefficient spectra) for the thin-film materials. Figure 2 shows a practical demonstration of the use of this software to fit the transmission spectrum and also to determine the optical absorption coefficient for a highly Bismuth-substituted metal-doped iron garnet thin film. Figure 2(a) shows the peak-to-peak fitting of the measured transmission spectra of an as-deposited $684 \mathrm{~nm}$ garnet layer of composition type $\mathrm{Bi}_{0.9} \mathrm{Lu}_{1.85} \mathrm{Y}_{0.25} \mathrm{Fe}_{4.0} \mathrm{Ga}_{1} \mathrm{O}_{12}$, where zero absorption coefficient was considered for the modelled transmission spectrum. Figure 2(b) shows the derived absorption coefficients of the same garnet layer. And figure 2(c) shows the transmission spectrum fitting of a 1310nm as-deposited garnet sample of the same composition type, where the derived absorption coefficients were considered during the simulation. 
Encyclopedia

MDPI
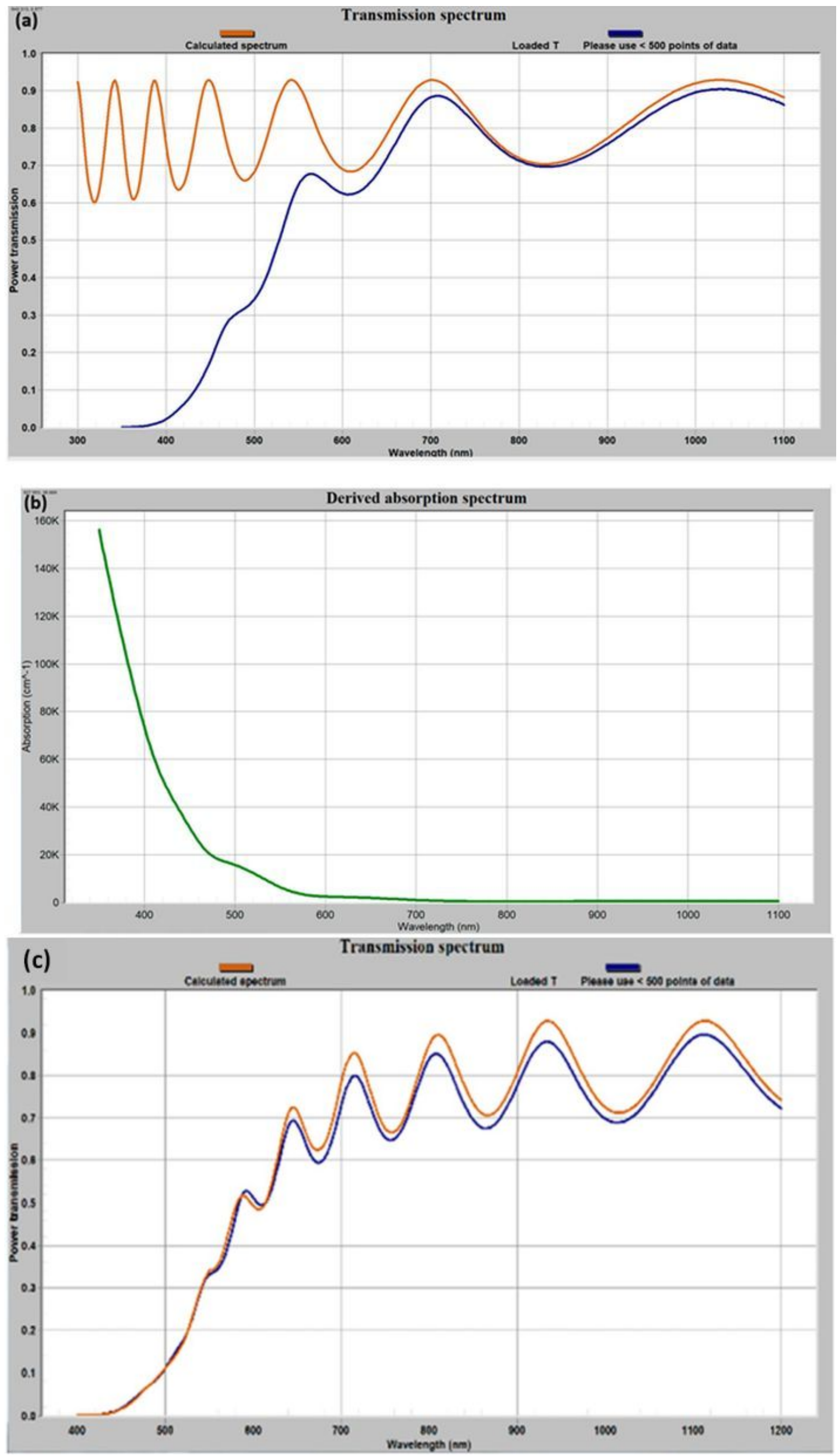

Encyclopedia 2020 doi: 10.32545/encyclopedia202004.0003.v4

3 
Figure 2. Magneto-photonic crystal (MPC) software fitted transmission spectra and the iterative (bisection algorithmassisted) fitting of the absorption coefficient spectral dependency and the required pre-fitting of film thickness through matching transmission peak features.

In order to understand the suitability and also to validate the calculation process results using this free (available online, https://doi.org/10.3390/technologies7030049) and easy to install and use software, several multi-defect multilayer MPC design, characterization, and optimisation examples have been trialled, used for illustration purposes. Figure 3 shows the result of an example run of MPC optimization software to reproduce the flat-top MPC transmission and Faraday rotation spectral properties for a four-defect MPC design known in the specialist literature.

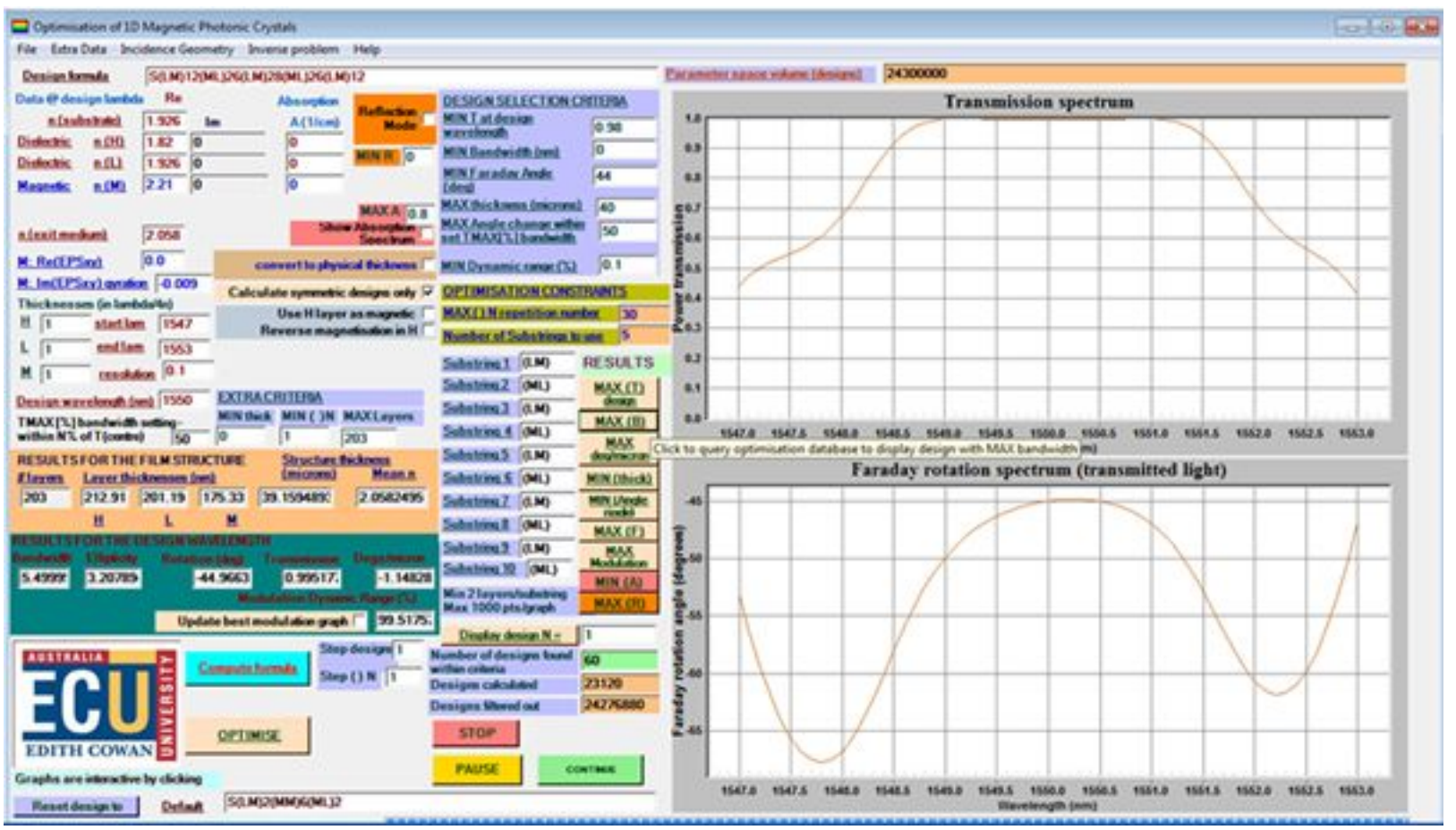

Figure 3. Result of reproduction of an optimized MPC design featuring "flat-top" response by using this new MPC optimization algorithm.

This MPC software package allows running the computational modelling of the optical spectral properties of various dielectrics-based generic single- and multilayer semitransparent thin films. Other additional program features of this software are the tools for fitting of the experimentally-measured transmission or reflection spectra to the theoretical models, and the films physical thickness data recovery, if detailed refractive index information is available. Fitting of the absorption coefficient spectra in absorbing material layers is also possible, using an automated algorithm reliant on the data for the measured transmission spectrum, refractive index spectrum, and physical thickness. A number of magnetooptic garnet material-related datasets are also available from the program installation directory, after the installation has been performed.

\section{References}

1. Mikhail Vasiliev; Kamal Alameh; Mohammad Nur-E-Alam; Nur- E- Alam; Analysis, Optimization, and Characterization of Magnetic Photonic Crystal Structures and Thin-Film Material Layers. Technologies 2019, 7, 49,

10.3390/technologies7030049.

\section{Keywords}


1D magnetic photonic crystals; multilayer film modeling; modeling of Faraday rotation spectra; MPC optimization; exhaustive computation; materials characterization

(c) (i) (C) 2020 by the author(s). Distribute under aCreative Commans CC BY license 